\title{
De vita et studiis viri septuagenarii Adalberti Stawiszyński commentatio cum versibus elegiacis in honorem eius adnexis
}

Si quis Latino sermone honorari meruit, certo vir clarissimus Adalbertus Stawiszyński qui annum aetatis septuagesimum nuperrime complevit. Inter omnes enim qui ad studia patristica in Polonia promovenda plurimum contulerunt, ille eximium praecipuumque obtinet locum.

„Quis est hic? et laudabimus eum - ait Ecclesiasticus $(31,9)$ - fecit enim mirabilia in vita sua". Nolite putare, benevolentissimi lectores, me abusum esse istis ex Ecclesiastico libro depromptis. Namque vere mirabilia fecit! Quamvis neque in artibus liberalibus studia absolverit nec gradum academicum in sacra theologia obtinuerit, inter eos qui nunc sunt, Patrum Ecclesiae cultores, eminet maximeque admirabilis in Polonia extat. Estne id verum? Ita sane et si parum vobis videtur, quod magis admiremini adiciam. Adalbertus nec Graecorum nec Latinorum auctorum linguas accuratissime umquam scrutatus est, ut mos est aliorum theologiae doctorum, nam exactis scientiis studebat! Agnoscite igitur virum studiis patristicis deditum praeclareque meritum atque honore dignum. Haud facile tamen est, etsi velim, clarissimum virum laudibus dignis ornare. Attamen licet brevi hac commentatione omnia penitus enarrari non possint, Adalbertum nostrum, optimi Patrum amatores, vobis ante oculos propono.

De his narraturus primum quidquid de puerili et iuvenili Adalberti aetate dicam, deinde ingenarii machinariae artis industriam exponam, tum nonnulla de actuositate virtutibusque eximiis quibus in societate civili eminuit, adiciam, denique assiduum cultorem fautoremque strenuum Patrum sanctorum laudabo.

Kalendis Ianuariis anno millesimo nongenetesimo quinquagesimo primo, matre Alicia et patre Francisco, domi parentum, ut mos illo tempore 
erat, Varsaviae, principali in urbe Poloniae, natus est. Cum in ecclesia paroeciali S. Sigismundi martyris, Burgundiorum regis, sita in hac urbis parte quae vulgo Bielany dicitur, sacro fonte ablutus esset, eo temporis - ut ipse scripsit - aevo Patrum feliciter occurrit. Puer annorum sex ludum litterarium, nomine Janusz Kusociński postea nuncupatum, frequentare coepit. Primis litterarum rudimentis acceptis, quarto et decimo aetatis anno, civibus lycei nomine Iosephi Martí dicto adscriptus est ibique anno millesimo nongentesimo duodeseptuagesimo examen maturitatis superavit. Eodem anno mense Martio ad studentes regimini communistarum obstantes adiit, quod quidem variarum operarum quae post aliquos annos in societate civili strenue ac diligenter acturus erat, quasi initium fuit. Iuvenis institutioni catholicae religionis apud ecclesiam paroecialem se tradidit, cuius testimonium, quod animadvertere expedit, ab ipso Em.mo ac Rev.mo D.no Cardinali Stephano Wyszyński, tunc Primate Poloniae, accepit. Ab anno millesimo nongentesimo duodeseptuagesimo ad millesimum nongentesimum septuagesimum quartum, studiis chimiae in Schola Polytechnica Varsaviensi, ubi beatae iam memoriae frater eius Andreas iisdem iam pridem incubuerat, nomen dedit, eo vel magis quod pater eius artis mechanicae ingenarius erat. Quibus feliciter peractis mense Martio anno millesimo nongentesimo septuagesimo quarto gradum magistri ingenarii in provincia catalysis heterogenae obtinuit.

Sed satis iam de puerili iuvenilique Adalberti aetate. Absoluto studiorum technicorum curriculo per unius anni spatium in officina vulgo Chemipan dicta chromatographiam gasiorum tractavit. Ab anno millesimo nongentesimo septuagesimo sexto ad millesimum nongentesimum octogesimum, examine ad doctoratum adipiscendum peracto, in Schola Polytechnica Varsaviensi studiis incubuit, deinde technicus unum per annum ibidem opus fecit. Attamen eadem in schola, a mense Augusto anno millesimo nongentesimo octogesimo primo, ob causas politicas non potuit diutius operari, propter quod doctoralem dissertationem interrupit scribendam. Tunc in officina chimica Libella dicta laboriosus operae brevissime impendit, nam paucis iam diebus post statum belli a regimine communistarum in Polonia proclamatum statim expulsus est. Duobus mensibus elapsis in officina Inco dicta laborans, medicaminum seu cosmeticorum producendorum technologiam didicit. Secundo dimidio anni millesimi nongentesimi octogesimi tertii opus reperit in societate Suecici negotiatoris Ingemari Svenson, Scan NS dicta, in qua ad mensem Septembrem anni millesimi nongentesimi undenonagesimi, primum technologus primarius, deinde vicedirector laboravit. Post comitia parlamentaria anno millesimo nongente- 
simo undenonagesimo acta consociationem propriam Quartz S.A. dictam cum tribus sociis fundavit ad producendam aquam oxygenii in industria cosmetica necessariam. Post arduum laborem, duobus annis praeteriectis, meliora tempora venerunt. In consociatione negotiorum, quam Adalbertus olim fundaverat, iam triginta operarii laborabant, sed ille ipse labore longo defessus, re diligenter perpensa, die secunda Aprilis anno bis millesimo nono mercium partes debitas sibi venumdedit et perendino die in regionem Seinensem profectus est.

Nec est silentio praetereunda erga patriam clarissimi viri pietas. Adalbertus, quamquam socialismum commutabilem vel reformabilem ordinem primo censebat, omnem spem cito perdidit. Ad hoc vim habuerunt multae res gestae et quidem epistula episcoporum Poloniae ad episcopos Germaniae anno millesimo nongentesimo sexagesimo quinto missa, deinde obtrectationes et calumniae erga Primatem Poloniae, iuvenum rebellio, cui Adalbertus interfuit, anno millesimo nongentesimo duodeseptuagesimo exorta, de qua supra iam dictum est, invasio exercituum sovieticorum in Cechoslovaciam mense Augusto eodem anno facta, caedes operariorum in litore Maris Baltici mense Decembri anno millesimo nongentesimo septuagesimo confecta, operariorum rebellio anno millesimo nongentesimo septuagesimo sexto exorta, deinde Em.mi D.ni Cardinalis Caroli Wojtyła in Summum Pontificem anno millesimo nongentesimo septuagesimo octavo electio, quae tandem spes maxima omnibus visa est, primum eiusdem Pontificis apostolicum iter in Poloniam anno sequenti factum. Eodem anno Adalbertus sociam operam navare coepit in Consilio pro Operariis Defendendis constituto, seu ephemerides vel cartulas clam editas distribuens seu cum viris qui systemati communistico resistebant conversans. Sodalis ordinarius liberi collegii opificum vulgari nomine Solidarietatis nuncupati, curator negotiorum in officinis in secundum conventum eiusdem Solidarietatis in regione Masoviae agentis delegatus est. Qui opus suum post proclamationem status belli in Polonia nec levis nec timidus neglexit, verum etiam tenax et assiduus prosequendum curavit. Et licet non locus sit ut de singulis loquamur, nunc, optimi lectores, eorum quae studiosissime ac summa cum industria illo tempore egit, aliquot exempla quasi raptim mecum percurratis.

Anno nempe millesimo nongentesimo octogesimo secundo comitatum contradictionis collegii Solidarietatis in meridiana parte Varsaviensis palatinatus acturum ipse ordinavit. Eodem comitatu curante varia scripta, diaria, cartulae, libri in clandestina officina typis edebantur. Eorum qui in carceribus detenti sunt familiis auxilia praebebantur, operistitia et demon- 
strationes publicae ordinabantur. Maio et Iunio mensibus anno millesimo nongentesimo octogesimo secundo arcanam societatem Armeniam nuncupatam instituit, quam septem per annos moderatus est. Cuius inter multa et clara opera enumerandum est clandestinum consilium publicae opinioni explorandae institutum atque emissoriorum radiophonicorum et televisificorum, tum minorum tum maiorum constructio. Totam actuositatem clandestinam explevit anno millesimo nongentesimo undenonagesimo, post comitia mense Iunio in Polonia habita, paucis autem mensibus post a vita publica se omnino removit.

Quod ad libertatem recuperandam vir civilibus artibus praestans idemque patriae amans plurimum contulisset, ab auctoritatibus publicis Polonorum variis insignibus honestatus est: anno bis millesimo insigni De cultu civili meritus dicto a Primo Ministro Consilii Rei Publicae tradito, anno bis millesimo decimo Cruce Commandaria Insignis Poloniae Restitutae dicta a Praeside Rei Publicae tradita, anno bis millesimo decimo quarto Insigni honorario de Varsavia meritus nuncupato, anno bis millesimo undevicesimo Cruce Libertatis et Solidarietatis dicta item ab Praeside Rei Publicae collata.

Praetereo, ne in scribendo longior sim, multas et alias virtutes aliaque merita quibus vir clarissimus in rebus publicis enituit, quae tamen memoravi ad praesens sufficiant. Transeamus nunc ad Patrum amatorem et si non est vobis molestum, diutius hic commoremur. Quo modo, rogabitis, Patres primum convenit. Quo vitae temporis puncto? Legatis, quaeso, quae ipse scripserit. „Eram aetate annorum fortasse viginti, nec plurima audivi seu de Patribus seu de primo Christianorum aevo. Martyres, edictum Mediolanense, Concilium Nicaenum, Hieronymus scripturarum translator, Augustinus magnus theologus, Gregorius Magnus papa magnus... Nihil amplius... Anno fere septuagesimo secundo, multos libros lectitans, subito obstupui, conieci nam oculos in nomina Gregorii Nysseni, Gregorii Nazianzeni, Cyrilli Alexandrini, Nestorii, Theodoreti, cuiusdam Origenis et aliorum... Obstupui et puduit me ignorantiae meae. Atqui - ut catholicus - examen maturitatis ego superavi! Tunc in bibliopolium profectus duos libros de Patribus emi, alterum Patres Ecclesiae inscriptum Iohannis Campenhauseni et alterum, Epistulas scilicet Basilii Magni. Emi, perlegi, imbutus sum. Spiritus vere - ut aiunt - flat ubi vult. Quod tunc factum est quodque porro fit, quinquaginta sane annis elapsis, aliter explanare nequeo. Postea alios libro emi, De fide et De officiis ministrorum Ambrosii Mediolanensis, Epistulas Hieronymi, De Trinitate Aurelii Augustini, denique Lexicon Litterarum Primi Christianorum Aevi, nuperrime in lucem 
prolatum. O felicem casum! Tunc quidem tantas litterarum Christianarum divitias paulisper saltem meo comprehendi ingenio!

Quid amplius? Eo quoque tempore, cum ad magistri gradum assequendum dissertationem scribebam, feliciter accidit ut in sede catalysis opus suum faceret vir doctus Casimirus Starowieyski. Quem occasione oblata interrogavi utrum sciret Marcum Starowieyski. - Hic frater meus est - respondit. Et sic post paucum tempus novi virum doctissimum et reverendissimum, qui in bibliothecam patristicam, tunc apud viam Dewajtis sitam, benigne me invitavit. Brevi tempore novi alios viros, patrologiae peritos, Rev.dum Patrem Aemilium Stanula et Rev.dum D.num Vincentium Myszor. Alius vir doctus, Rev.dus D.nus Stanislaus Longosz, ad conventum patristicum Lublinense me invitavit et sic omnia coepta sunt. Ex eo tempore Patres me comitati sunt. Quorum scripta, dum autoreda vel tramine vehebar, longas per horas pervolutabam, ita ut in hac assidua conversatione perdifficili illo tempore solamen invenerim et gaudium. Non solum tamen opera Patrum legebam, sed etiam bibliothecam comparavi, in qua nunc sunt mihi plus quam tria milia voluminum, omnes fere operum Patrum in Polonicum versiones, scriptorum antiquorum tum Graecorum tum Romanorum tum orientalium collectio et alia multa. Cum autem bibliographiis, quae versionibus Polonicis additae sunt, nonnullas lacunas vel defectus inesse animadvertissem, novae bibliographiae patristicae parandae cepi consilium. O me miserum! Quod paucos per menses me facturum putabam, per longos octo annos facere debui! Exeunte anno bis millesimo quinto opus vitae meae feliciter in lucem prolatum est, Bibliographia patristica annorum 1901-2004 inscriptum. Quid nunc? Novum opus cepi, bibliographiam textuum antiquorum qui in Polonicum translati sunt, a Homero scilicet ad Simplicianum. Labor non deerit mihi”.

Haec quae supra ipse Adalbertus. Habetis itaque, optimi lectores, unde apud eum sit tantus amor Patrum, tanta erga Patres voluntas. Nunc de iis dicam quae scripsit, collegit, recensuit, examinavit, indicibus auxit, quae edenda curavit.

Quae inter primum enumerandi sunt duo libri eique maximi sane momenti, unus de quo ipse Adalbertus fecit mentionem, bibliographiam patristicam amplectens, completam et optime congestam, collectionem scilicet textuum Christianorum auctorum primi millennii in Polonorum sermonem versorum, qui inter annum millesimum nongentesimum primum et bis millesimum quartum in libris, anthologiis, variis articulis typis excusi sunt. Qui liber bis editus est apud officinam Tiniciensem, primum anno bis millesimo quinto et iterum, annis octo post. Tribus tamen post annis 
novum Bibliographiae patristicae volumen idque revisum, auctum et correctum eadem in Tiniciensi officina in lucem prolatum est. His adde Novum Lexicon Litterarum Primi Christianorum Aevi, recognitum et amplificatum, quod adiuvante Adalberto Stawiszyński nunc accuratissime exscribi Marcus Starowieyski curavit, qui primam eiusdem editionem cum doctissimo piae iam memoriae Ioanne Maria Szymusiak, multis annis ante diligentissime exaravit. Ad hanc alteram eiusdem lexiconis editionem indices ipse Adalbertus accuratissime addidit. Qua claruit autem assiduitas efficit ut in scribendis dissertationibus viris doctis, theologiae Patrum peritis, conspicuum saepius ac saepius adiumentum ferat. Cum Ioanne Iluk, professore Gedanensi, varias dissertationes et articulos Rev.di D.ni Stanislai Longosz, clarissimi veterum Patrum indagatoris, iam pridem vulgatos, selegit et accuravit. Deinde proximis duobus annis ad quattuor alios libros indices exaravit. Non possumus omittere quae composuit quaeque in commentario periodico Vox Patrum inscripto repertoria bibliographica vulgavit, libros quos existimavit et aliquot alia opuscula, quae ille solus vel cum aliis accurate exscripsit. Neque quisquam miretur hoc tempore per paginas interretiales commentationes suas, existimationes scilicet librorum qui Patrum aetatem spectent, Adalbertum pergere vulgandas.

Redeamus denique ad Adalberti verba, quibus de sui ipsius vita sic nuperrime narravit: „Ab eo tempore quo in Augustoviensi regione inhabitavi, duodecim transierunt anni. Plus quam decem iam annos habito in pago v.d. Stanowisko, quem vix triginta homines incolunt quique apud ripam fluminis dicti Marycha situs est. Domus nostra in rupe supra flumen aedificata, ultima est et in meridiem spectat. Ipsum flumen autem inter Poloniam et Lithuaniam exstat confinium, itaque quoties de fenestra prospicio, Lithuaniam videre possum. Habeo quoque domum hospitalem in qua bibliothecam meam posui. Vicinus meus unius chilometri spatio distat, taberna alimentaria aestate chilometrorum septem, hieme autem tredecim. In oppido Sejny dicto eoque pulchro et venusto basilicam minorem, arte quam Barocam nuncupant, constructam una cum vetere monasterio Ordinis S. Dominici visere poteris necnon deversorias tabernas et popinas, etiam Lithuanicas, invenies, valetudinarium, argentariam, ambulatorium, notarii officium, vigilum publicorum stationem et excubitorium militum in confinio factum... Omnia ergo quae ad vivendum necessaria sunt. Quod ad me attinet, qui hunc pagum antea inhabitabant, me amaverunt. Uxor mea Margarita (quam potius Margaritulam dico) scultetus pagi nostri iterum electa est. Quid mihi faciendum? Habet potestatem. Regio nostra ait - vasta est et deserta. Hic alcem quam homines facilius invenies. In 
silva Augustoviensi vivunt castores et nyctyreutes, lupi et martes, cervi et lepores, tetraones et falcones, ciconiae nigrae et grues, lyncem quoque vidi atque parvus bisontum grex peregre has in silvas approperavit. Quid amplius? Bene valeo in hac solitudine. Attamen non solus sum. Mecum est Margaritula, coniunx fidelissima, patiens, sollicita, attenta. Sciat itaque gratum animum meum".

Adalberte, vir colendissime! Divitiae diffluunt, vires minuuntur, fugaces $[. .$.$] labuntur anni. Tibi, viro septuagenario, ex animo gratulamur, quod$ maximo studio atque animi impetu doctrinam Patrum inter omnes fovendam curare et colere pergis. Tibi quidem, sincera animi voluntate optamus et ominamur ut Deo opitulante vivas, floreas, crescas.

Adalberto vita! Bene meritus! Bene dignus!

\section{Versus elegiaci in honorem Adalberti Stawiszyński, viri bene meriti}

Terdecies lustris quinos feliciter annos

Nunc modo consertos dat tibi Celsithronus.

Vir sapiens semper, moderatus, pacis amicus:

Et tibi concivium sedula cura fuit.

Statio tunc latuit quae phonica nuncupabatur,

Ad patriae sedes libera verba ferens.

Libertatis amans, maiora pericula cernis,

Nunc habitus dignus optimis et meritis.

Impiger, assiduus, gnavus, industrius: exhinc

Dilexisti Patres quos fide, corde colis.

Colligis et codicos, eorum dulcis amator,

Litteras Ecclesiae crescere laude facis.

Varsaviae natus, nunc in regione remota

Mansio grata sita est partibus in Boreae.

Fortunata domus! Silvas miraris et undas

Quas crebro stringit frigida bruma gelu.

Liquitur ut glacies, ver almum ridet in agris

Clangoreque gruis prata propinqua sonant.

Sumite gaudentes quae misi carmina parva

Et faveat vobis dans meliora Deus.

Gaude et tu felix, Margarita optima coniunx

Quae tuis officiis tecta laremque paras. 